\title{
Claves taxonómicas, basadas en la morfología de la aleta caudal, para la determinación de tiburones (Chondrichthyes; Elasmobranchii) de las costas de Chile
}

Taxonomic keys based on the morphology of the caudal fin, for the sharks identification (Chondrichthyes; Elasmobranchii) from the Chilean coasts

\author{
Sylvia Sáez ${ }^{1}$, Julio Lamilla1 ${ }^{1}$ y Germán Pequeño ${ }^{1}$
}

\begin{abstract}
${ }^{1}$ Instituto de Ciencias Marinas y Limnológicas, Laboratorio de Elasmobranquios (ELASMOLAB), Universidad Austral de Chile, Casilla 567, Valdivia, Chile.sylvia.saez@gmail.com

Abstract.- The role of shark's caudal fin has been studied from a morpho-functional point of view, in which it is established that participates in the fish locomotion, as well as in the prey capture by Alopiidae species. However, this trait has been scarcely used in taxonomic studies. Given the interspecific variation of this structure, it is hypothesized that might be used as a morphologic character for the species identification. Because of this, the preparations of taxonomic keys based on morphological specific characteristics of the caudal fin are needed, mainly when partially destroyed specimens are studied. This study proposes taxonomic keys for the 58 sharks species registered in Chile. These keys were made with the aim to be used in diagnosis and specific taxonomic identification of this fish group, as a complement or as an independent way, with relation to the already existent ones.
\end{abstract}

Key words: Taxonomy, cartilaginous fishes, caudal peduncle, dorsal margin, preventral margin

Resumen.- El rol de la aleta caudal en tiburones, ha sido estudiado desde un enfoque morfo-funcional, desde el punto de vista de la locomoción del individuo como en la captura de alimento por Alopiidae. Sin embargo, este carácter morfológico de tiburones ha sido escasamente empleado en estudios taxonómicos. Dada la variación interespecífica de este carácter, es que podría utilizarse como carácter morfológico alternativo en la determinación de especies. Por ello, es necesaria la elaboración de claves taxonómicas con características morfológicas específicas de la aleta caudal, especialmente cuando se trabajan con ejemplares incompletos. Este estudio propone claves taxonómicas para las 58 especies de tiburones registradas hasta hoy en Chile, con la finalidad de ser empleadas en la diagnosis y determinación taxonómica específica de este grupo de peces, como complemento o de manera independiente a las claves ya existentes.

Palabras clave: Taxonomía, peces cartilaginosos, pedúnculo caudal, margen dorsal, margen preventral

\section{INTRODUCCIÓN}

La aleta caudal de los tiburones presenta una amplia variedad de componentes morfológicos como: margen dorsal, lóbulo dorsal, lóbulo terminal, extremo posterior, margen terminal, extremo subterminal, margen subterminal, muesca subterminal, margen postventral, entre otros (Fig. 1a), que son de gran utilidad para ser empleados como caracteres taxonómicos (Compagno et al. 2005, Hernández et al. 2009). Tales componentes morfológicos han sido estudiados desde un enfoque morfo-funcional, es decir, describiendo la utilidad de la aleta caudal tanto para la locomoción del individuo, como para la destreza en los movimientos que permiten la captura de alimento, e.g., Alopias vulpinus, donde utiliza el lóbulo dorsal de la aleta caudal para aturdir a la presa antes de ser capturada, cumpliendo funciones de persecución e inmovilización a la presa (Stewart \& Simaner 1977, Aalbers et al. 2010, Reiss \& Bonnan 2010). Sin embargo, pese a la variedad de los componentes morfológicos antes mencionados, la aleta caudal de los tiburones es empleada escasamente en las claves de determinación taxonómica en los condrictios, formando sólo parte del conjunto de caracteres diagnósticos de la morfología corporal externa en este grupo de peces (Pequeño 1977, 1979 a, b; Compagno 1984 a, b; Lloris \& Rucabado 1991, Brito 2004 a, b; Lamilla \& Bustamante 2005, Valenzuela et al. 2008, Sáez et al. 2010). Es frecuente encontrar claves donde la combinación de caracteres externos tales como: forma y 
color del cuerpo, ausencia o presencia de quilla caudal, entre otros, sirven para ser aplicadas a un ejemplar completo, sin embargo, los individuos capturados en las pesquerías no siempre se encuentran en buen estado de conservación, lo que dificulta la determinación de las especies. En estos casos, la morfología de la aleta caudal (Fig. 1a) podría ser utilizada como herramienta para la determinación taxonómica de órdenes, familias, géneros y especies. El objetivo del presente estudio fue elaborar una clave taxonómica complementaria con base en los distintos componentes morfológicos de la aleta caudal de los tiburones. En este sentido, se provee una herramienta taxonómica complementaria para ser utilizada en la determinación de las 58 especies de tiburones presentes en aguas de Chile.

\section{Materiales Y MÉTODOS}

Para la elaboración de las claves taxonómicas se utilizaron las imágenes o esquemas de la aleta caudal (Compagno 1984 a, b; Compagno et al. 2005) de 32 especies registradas en aguas de Chile: Heptranchias perlo (Bonnaterre, 1788), Chlamydoselachus anguineus Garman, 1884; Rhincodon typus Smith, 1828; Rhina armata Philippi, 1887; Carcharodon carcharias (Linnaeus, 1758); Cetorhinus maximus (Gunnerus, 1765); Cephaloscyllium ventriosum (Garman, 1880); Cephalurus cephalus Gilbert, 1892; Mustelus whineyi Chirichigno, 1973; Triakis maculata Kner \& Steindachner, 1867; Carcharhinus falciformis (Müller \& Henle, 1839); Carcharhinus obscurus (Lesueur, 1818); Sphyrna lewini (Griffith \& Smith, 1834); Centrophorus squamosus (Bonnaterre, 1788); Euprotomicrus bispinatus (Quoy \& Gaimard, 1824); Heteroscymnoides marleyi Fowler, 134; Isistius brasiliensis (Quoy \& Gaimard, 1824); Mollisquama parini Dolganov, 1984; Echinorhinus cookei Pietschmann, 1928; Centroscyllium nigrum Garman, 1899; Etmopterus brachyurus Smith \& Radcliffe, 1912; E. litvinovi Parin \& Kotlyar, 1990; E. lucifer Jordan \& Snyder, 1902; E. pycnolepis Kotlyar, 1990; E. pusillus (Lowe, 1839); E. unicolor (Engelhardt, 1912); E. villosus Gilbert, 1905; Centroscymnus owstonii Garman, 1906; Scymnodalatias oligodon Kukuyev \& Konovalenko, 1988; Somniosus pacifus Bigelow \& Schroeder, 1944; Squalus blainville (Risso, 1827); Squalus mitsukurii Jordan \& Snyder, 1903.

Adicionalmente, se utilizaron dibujos de las 27 especies restantes, que pertenecen a la colección de docencia de Peces Elasmobranquios del Instituto de Ciencias Marinas y Limnológicas de la Universidad Austral de Chile. Las especies fueron las siguientes: Centroscyllium granulatum Günther, 1887; Zameus squamulosus Günther, 1887; Hexanchus griseus (Bonnaterre, 1788); Notorhynchus cepedianus (Péron, 1807); Pseudocarcharias kamoharai (Matsubara, 1936); Isurus oxyrinchus Rafinesque, 1809; I. paucus Guitart Manday, 1966; Lamna nasus (Bonnaterre, 1788); Alopias superciliosus (Lowe, 1841); A. vulpinus (Bonnaterre, 1788); Apristurus brunneus (Gilbert, 1829); A. nasutus De Buen, 1959; Bythaelurus canescens (Günther, 1878); Schroederichthys bivius (Müller \& Henle, 1838); S. chilensis (Guichenot, 1848); Galeorhinus galeus (Linnaeus, 1758); Mustelus mento Cope, 1877; Carcharhinus galapagensis (Snodgrass \& Heller, 1905); Prionace glauca (Linnaeus, 1758); Sphyrna zygaena (Linnaeus, 1758); Deania calcea (Lowe, 1839); Aculeola nigra De Buen, 1959; Etmopterus granulosus (Günther, 1878); C. crepidater (Barbosa du Bocage \& de Brito Capello, 1864); Centroscymnus macracanthus Regan, 1906 y Squalus acanthias Linnaeus, 1758.

Con el propósito de evaluar la variabilidad intraespecífica con relación a la morfología de la aleta caudal, se utilizaron 5 individuos por especie, tanto en los ejemplares de colección como en diferentes ilustraciones, seleccionándose el morfotipo caudal más frecuente.

La metodología de clasificación taxonómica utilizada en la preparación de las claves, fue la propuesta por Compagno (1999) y Eschmeyer (2011). ${ }^{1}$

Para facilitar el manejo de la terminología de los caracteres taxonómicos que acompaña las partes de la aleta caudal, se ilustra un esquema general (Fig. 1 a). Asimismo, fue medido el ángulo de inclinación de la aleta caudal, utilizando un transportador, proyectando una línea por el borde dorsal del pedúnculo caudal y su intersección con el extremo posterior de la aleta caudal (Fig. 1 b).

\section{Resultados}

A continuación se presentan 2 tipos de claves sucesivas en orden jerárquico: una clave inicial, que permite reconocer 4 órdenes de tiburones distribuidos en aguas chilenas. A partir de dicha clave, se desglosan otras 4 claves para el reconocimiento de las especies dentro de los órdenes Hexanchiformes, Lamniformes, Carcharhiniformes y Squaliformes.

En Orectolobiformes y Squatiniformes, por tratarse de taxa monotípicos en aguas chilenas, las características de la aleta caudal se presentan en la misma clave de órdenes.

${ }^{1}$ Eschmeyer W. 2011. Catalog of fishes. California Academy of Sciences, San Francisco. [on line] <http//: www. calacademy.org> 


\section{Clave taxonómica para la determinación de órdenes}

1 (2) Lóbulo dorsal más pequeño que el ventral.....Squatiniformes: Squatinidae: Ángulo de inclinación de la aleta de $34^{\circ}$. Margen terminal y subterminal ausente. Margen postventral inferior más largo que el superior

2 (1) Lóbulo dorsal más grande que el ventral

Rhina armata (Fig. 2 f).

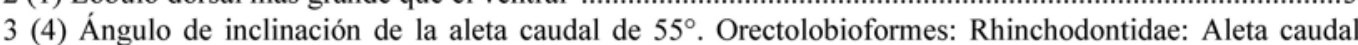
semilunada, sin muescas, con un único patrón de manchas blancas sobre un fondo oscuro. Margen postventral inferior más corto que el superior.

4 (3) Ángulo de inclinación de la aleta caudal menor de $55^{\circ}$ Rhincodon typus (Fig. 2 e).

5 (6) Ángulo de inclinación de la aleta caudal entre $10^{\circ}$ y $15^{\circ}$ Hexanchiformes (Clave 1).

6 (5) Ángulo de inclinación de la aleta caudal mayor a $15^{\circ}$

7 (8) Lóbulo dorsal grande y el ventral casi del mismo tamaño (excepto en Pseudocarchariidae) dando el aspecto de cola semilunada (excepto Alopiidae). Ángulo de inclinación de la aleta caudal entre $19^{\circ}$ y $50^{\circ}$

.Lamniformes (Clave 2).

8 (7) Lóbulo dorsal siempre más grande que el ventral

9 (10) Muesca posterior muy desarrollada si está presente. Ángulo de inclinación de la aleta caudal entre $11^{\circ}$ y $45^{\circ}$ Carcharhiniformes (Clave 3).

10 (9) Muesca posterior poco desarrollada si está presente. Ángulo de inclinación de la aleta caudal entre $12^{\circ}$ y $50^{\circ}$ Squaliformes (Clave 4).
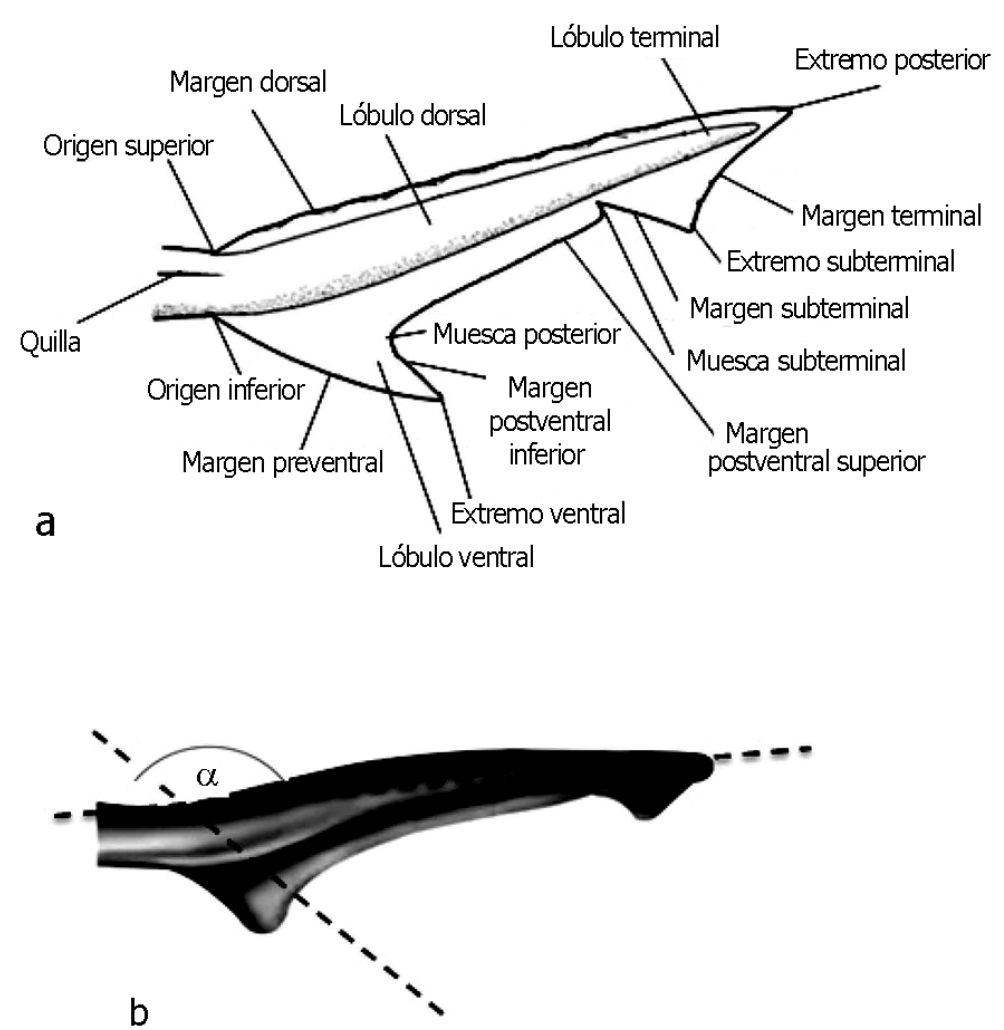

Figura 1. a) Esquema general de la aleta caudal y su nomenclatura (modificado de Compagno et al. 2005). b) Ejemplo de la medición (igual a 15) de la aleta caudal en Hexanchus griseus / a) General scheme of the caudal fin and its nomenclature (modified from Compagno et al. 2005). b) Example of measurement (equals to 150) of the caudal fin in Hexanchus griseus 


\section{Clave 1. Clave taxonómica para la determinación de familias, géneros y especies del orden Hexanchiformes}

1 (2) Márgenes terminal y subterminal presentes. Margen postventral inferior presente. Ángulo de inclinación de la aleta caudal entre $10^{\circ}$ y $15^{\circ}$ Hexanchidae: 3 2 (1) Márgenes terminal y subterminal ausentes. Margen postventral inferior ausente. Ángulo de inclinación de la aleta caudal de $12^{\circ}$ Chlamydoselachiidae: Chlamydoselachus anguineus (Fig. 2 d). 3 (4) Muesca subterminal y muesca posterior desarrolladas. Ángulo de inclinación de la aleta caudal menor de $15^{\circ}$ 5 4 (3) Sin muesca subterminal y muesca posterior no muy desarrollada. Ángulo de inclinación de la aleta caudales de $15^{\circ}$. Hexanchus griseus (Fig. 2 b). 5 (6) Margen terminal ancho, con extremo posterior dirigido hacia abajo. Lóbulo terminal ancho. Ángulo de inclinación de la aleta caudal de $10^{\circ}$ Heptranchias perlo (Fig. 2 a). 6 (5) Margen terminal angosto, con extremo posterior derecho. Lóbulo terminal angosto. Ángulo de inclinación de la aleta caudal de $14^{\circ}$ Notorynchus cepediamus (Fig. 2 c).

\section{Clave 2. Clave taxonómica para la determinación de familias, géneros y especies del orden Lamniformes}

1 (2) Lóbulo dorsal extremadamente desarrollado, respecto al ventral; su longitud más de seis veces la altura del lóbulo ventral. Lóbulo terminal angosto. Ángulo de inclinación de la aleta caudal entre $19^{\circ}$ y $28^{\circ}$ Alopiidae: 3 2 (1) Lóbulo dorsal más grande que el ventral, pero no excesivamente desarrollado; su longitud menos de seis veces la altura del lóbulo ventral. Lóbulo terminal ancho. Ángulo de inclinación de la aleta caudal menor de $28^{\circ}$

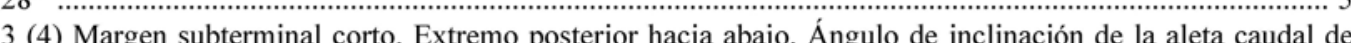
$28^{\circ}$ Alopias superciliosus (Fig. $3 \mathrm{~g}$ ).

4 (3) Sin margen subterminal. Extremo posterior derecho. Ángulo de inclinación de la aleta caudal de $19^{\circ}$ Alopias vulpinus (Fig. $3 \mathrm{~h}$ ).

5 (6) Margen postventral inferior corto. Margen terminal sinuoso. Ángulo de inclinación de la aleta caudalde $26^{\circ}$.............................................................. Pseudocarcharhiidae: Pseudocarcharias kamoharai (Fig. 3 a).

6 (5) Margen postventral inferior largo. Margen terminal recto. Ángulo de inclinación de la aleta caudal mayor a $26^{\circ}$

7 (8) Margen preventral recto. Ángulo de inclinación de la aleta caudal de $44^{\circ}$ Cetorhinidae: Cetorhinus maximus (Fig. 3 f).

8 (7) Margen preventral redondeado. Ángulo de inclinación de la aleta caudal entre $26^{\circ}$ y $53^{\circ}$

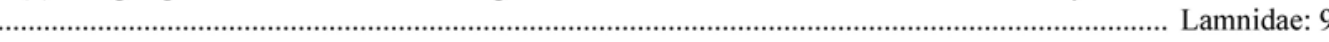

9 (10) Lóbulo dorsal más angosto que el ventral. Margen postventral inferior recto y angosto

10 (9) Lóbulo dorsal más ancho que el ventral. Margen postventral inferior sinuoso. Ángulo de inclinación de la aleta caudal de $46^{\circ}$ Lamna nasus (Fig. 3 e).

11 (12) Con muesca subterminal. Ángulo de inclinación de la aleta caudal entre $44^{\circ}$ y $53^{\circ}$............ Isurus: 13 12 (11) Sin muesca subterminal. Ángulo de inclinación de la aleta caudal de $41^{\circ}$

Carcharodon carcharias (Fig. 3 b).

13 (14) Margen terminal recto. Margen subterminal contenido tres veces en el margen terminal. Margen postventral inferior recto. Ángulo de inclinación de la aleta caudal de $44^{\circ}$ Isurus paucus (Fig. 3d). 14 (13) Margen terminal cóncavo. Margen subterminal contenido cuatro o más veces en el margen terminal. Margen post ventral inferior redondeado. Angulode inclinación dela aleta caudalde $53^{\circ}$.

I. oxyrinchus (Fig. $3 \mathrm{c}$ ). 


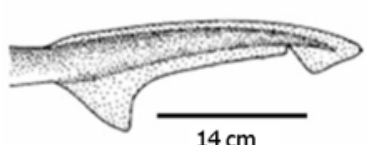

a

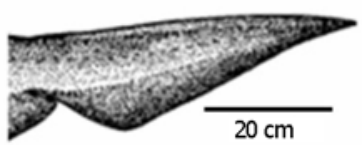

d

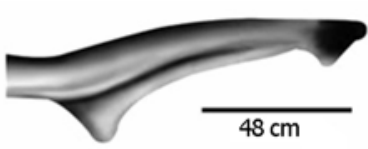

b

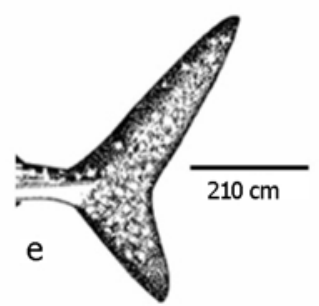

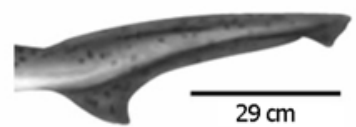

C

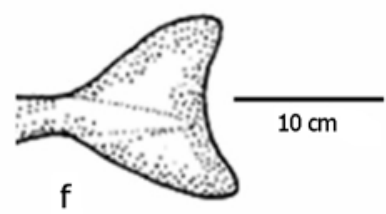

Figura 2. llustración de la aleta caudal de Hexanchiformes (a-d), Orectolobiformes (e) y Squatiniformes (f), indicando la longitud total (LT) de cada especie / Illustration of the Hexanchiformes (a-d), Orectolobiformes (e) and Squatiniformes (f) caudal fins, indicating the total body length of each species (TL)

Nombres científicos / Scientific names:

a) HEXANCHIDAE: Heptranchias perlo (Bonnaterre, 1788) LT = $139 \mathrm{~cm}$; b) Hexanchus griseus (Bonnaterre, 1788) LT = 482 cm, c) Notorynchus cepedianus (Pèron, 1807) LT $=290 \mathrm{~cm}$; d) CHLAMYDOSELACHIDAE: Chlamydoselachus anguineus Garman, 1884 LT = $196 \mathrm{~cm}$; e) RHINCODONTIDAE: Rhincodon typus Smith, 1828 LT = $2100 \mathrm{~cm}$; f) SQUATINIDAE: Rhina armata Philippi, 1887 LT = $103 \mathrm{~cm}$

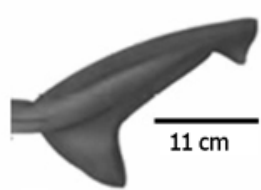

a

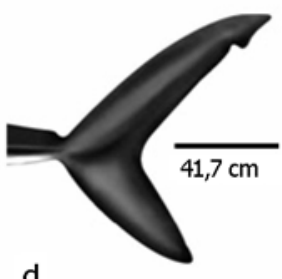

d

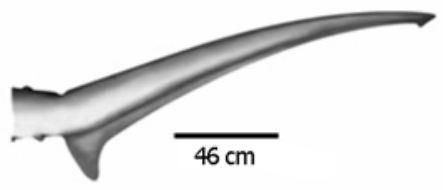

b
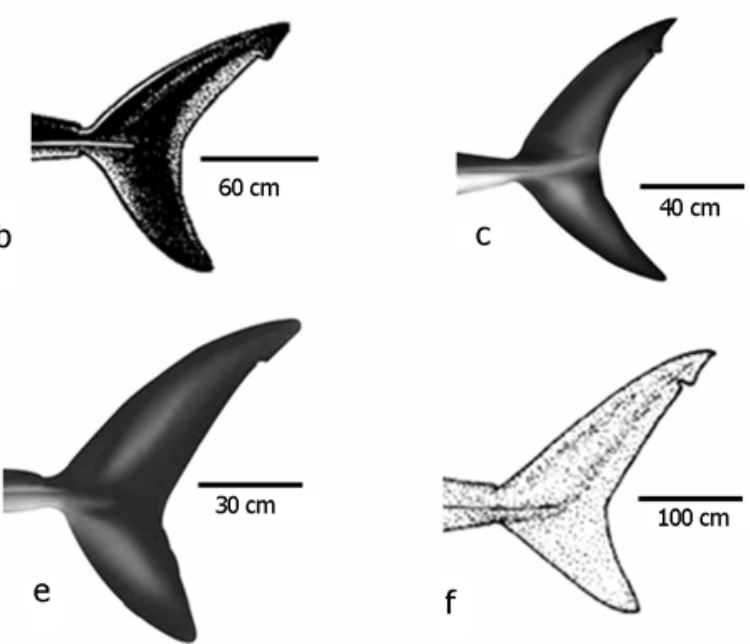

$\mathrm{h}$

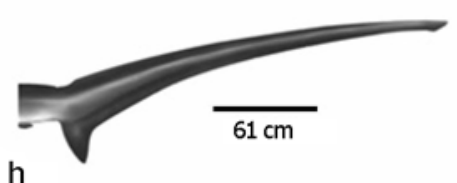

Figura 3. Ilustración de la aleta caudal de Lamniformes (a-h), indicando la longitud total (LT) de cada especie / Illustration of the Lamniformes caudal fin (a-h), indicating the maximum size of each species (TL)

Nombres científicos / Scientific names:

a) PSEUDOCARCHARIIDAE: Pseudocarcharias kamoharai (Matsubara, 1936) LT $=110 \mathrm{~cm}, \mathrm{~b}$ ) LAMNIDAE: Carcharodon carcharias (Linnaeus, 1758) LT = $600 \mathrm{~cm}$; c) Isurus oxyrinchus Rafinesque, $1809 \mathrm{LT}=400 \mathrm{~cm}$; d) Isurus paucus Guitart Manday, $1966 \mathrm{LT}=417 \mathrm{~cm}$; e) Lamna nasus (Bonnaterre, 1788) LT = $300 \mathrm{~cm}$; f) CETORHINIDAE: Cetorhinus maximus (Gunnerus, 1765) LT = $1000 \mathrm{~cm} ; \mathrm{g}$ ) ALOPIIDAE: Alopias superciliosus ( Lowe, 1841) LT $=460 \mathrm{~cm}$; h) Alopias vulpinus (Bonnaterre, 1788) $\mathrm{LT}=610 \mathrm{~cm}$ 
Clave 3. Clave taxonómica para la determinación de familias, géneros y especies del orden Carcharhiniformes

1 (2) Extremo subterminal puntiagudo. Con o sin margen postventral inferior 3

2 (1) Extremo subterminal redondeado. Sin margen postventral inferior. Ángulo de inclinación de la aleta caudal entre $10^{\circ}$ y $23^{\circ}$

3 (4) Ángulo de inclinación de la aleta caudal entre $16^{\circ}$ y $25^{\circ}$ Scyliorhinidae: 7

4 (3) Ángulo de inclinación de la aleta caudal mayor a $25^{\circ}$ Triakidae: 19

5 (6) Margen terminal recto y oblicuo o ligeramente cóncavo. Ángulo de inclinación de la aleta caudal entre $19^{\circ}$ y $45^{\circ}$

6 (5) Margen terminal cóncavo. Ángulo de inclinación de la aleta caudal entre $25^{\circ}$ y $32^{\circ}$

7 (8) Sin muesca subterminal. Ángulo de inclinación de la aleta caudal entre $12^{\circ}$ y $14^{\circ}$

8 (7) Con muesca subterminal. Ángulo de inclinación de la aleta caudal entre $10^{\circ}$ y $17^{\circ}$

Carcharhinidae: 25 Sphyrnidae: 31

9 (10) Margen postventral superior sinuoso. Ángulo de inclinación de la aleta caudal de $12^{\circ}$ Schroederichthys: 9

(1)
10 (9) Margen postventral superior recto. Ángulo de inclinación de la aleta caudal de $16^{\circ}$ Schroederichthys bivius (Fig. 4 f). Schroederichthys chilensis (Fig. 4 g). 11 (12) Margen terminal ancho, muesca subterminal notoria. Ángulo de inclinación de la aleta caudal de $16^{\circ}$

Cephaloscyllium ventriosum (Fig. 4 d). 12 (11) Margen terminal angosto, muesca subterminal poco profunda. Ángulo de inclinación de la aleta caudal mayor o igual a $16^{\circ}$

13 (14) Ángulo de inclinación de la aleta caudal de $18^{\circ}$

14 (13) Ángulo de inclinación de la aleta caudal de $16^{\circ}$

Cephalurus cephat

15 (16) Margen terminal con una muesca. Lóbulo ventral angosto. Ángulo de inclinación de la aleta caudal de $17^{\circ}$ Bythaelurus canescens (Fig. 4 c). 16 (15) Margen terminal liso. Lóbulo ventral ancho. Ángulo de inclinación de la aleta caudal menor a $17^{\circ} \ldots \ldots \ldots \ldots \ldots \ldots . . . . . . . . .17$ 17 (18) Margen postventral superior redondeado; extremo ventral aguzado. Ángulo de inclinación de la aleta caudal de $10^{\circ}$.............................................................................................................................. Apristurus bruneus (Fig. 4 a). 18 (17) Margen postventral superior sinuoso, extremo ventral redondeado. Ángulo de inclinación de la aleta caudal de $14^{\circ}$ ..... Apristurus nasutus (Fig. 4 b) 19 (20) Margen postventral inferior muy desarrollado y su tamaño contenido dos veces en el margen terminal. Extremo ventral redondeado. Ángulo de inclinación de la aleta caudal de $25^{\circ}$................................... Galeorhinus galeus (Fig. 4 h). 20 (19) Margen postventral inferior poco desarrollado (contenido menos de dos veces en el margen terminal) o ausente. Ángulo de inclinación de la aleta caudal menor a $25^{\circ}$.

21 (22) Margen terminal recto a ligeramente cóncavo. Margen postventral superior recto u ondulado. Extremo ventra redondeado. Ángulo de inclinación de la aleta caudal entre $13^{\circ}$ y $23^{\circ}$.......................................................... Mustelus: 23 22 (21) Margen terminal redondeado. Margen postventral superior sinuoso. Extremo ventral en punta. Ángulo de inclinación de la aleta caudal de $16^{\circ}$.......................................................................... Triakis maculata (Fig. 4 k). 23 (24) Margen terminal recto y oblicuo. Extremo ventral redondeado. Ángulo de inclinación de la aleta caudal de $13^{\circ}$ .. Mustelus mento (Fig. 4 i). 24 (23) Margen terminal levemente cóncavo. Extremo ventral puntiagudo. Ángulo de inclinación de la aleta caudal de $23^{\circ}$

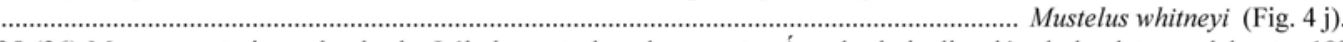
25 (26) Muesca posterior redondeada. Lóbulo ventral ancho y corto. Ángulo de inclinación de la aleta caudal entre $19^{\circ}$ y $45^{\circ}$ Carcharhinus: 27

26 (25) Muesca posterior recta. Lóbulo ventral angosto y alargado. Ángulo de inclinación de la aleta caudal de $25^{\circ}$

27 (28) Margen terminal levemente cóncavo. Ángulo de inclinación mayor a $19^{\circ}$ Prionace glauca (Fig. 4 ñ).

. 29 28 (27) Margen terminal recto. Ángulo de inclinación de la aleta caudal de $19^{\circ}$............ Carcharhinus falciformis (Fig. 4 l), 29 (30) Margen dorsal con una sinuosidad al llegar al extremo posterior, lóbulo terminal angosto. Ángulo de inclinación de la aleta caudal de $33^{\circ}$ Carcharhinus obscurus (Fig. 4 n). 30 (29) Margen dorsal recto, lóbulo terminal ancho. Ángulo de inclinación de la aleta caudal de $45^{\circ}$......

Carcharhinus galapagensis (Fig. $4 \mathrm{~m}$ ). 31 (32) Muesca subterminal poco profunda. Muesca posterior aguzada. Angulo de inclinación de la aleta caudal de $32^{\circ}$ Sphyrna lewini (Fig. 4 o). 32 (31) Muesca subterminal profunda. Muesca posterior redondeada. Ángulo de inclinación de la aleta caudal de $25^{\circ}$ Sphyrna zygaena (Fig. 4 p). 


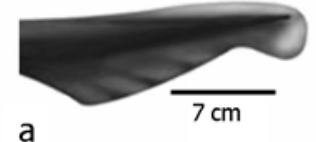

a

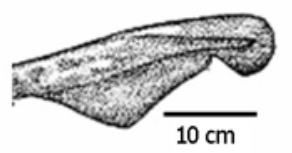

d
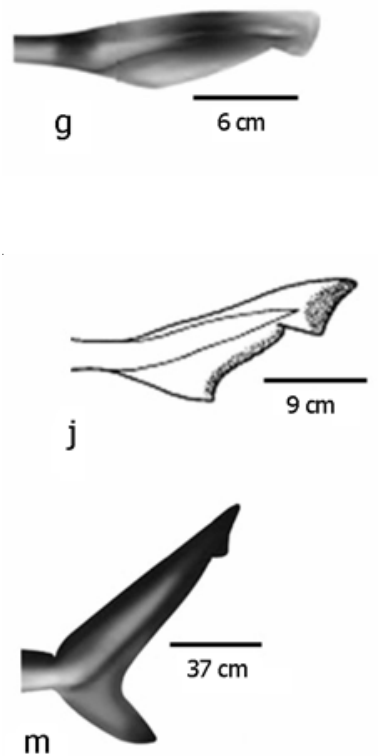

$\mathrm{m}$

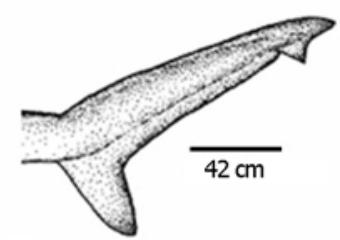

0

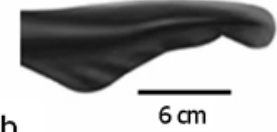

b

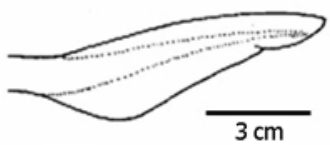

e
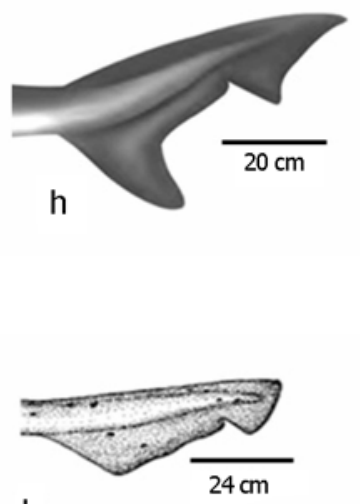

k

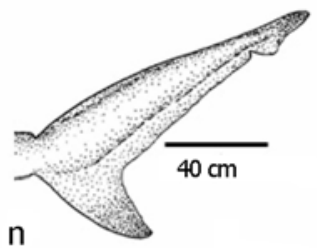

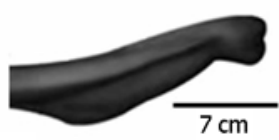

C
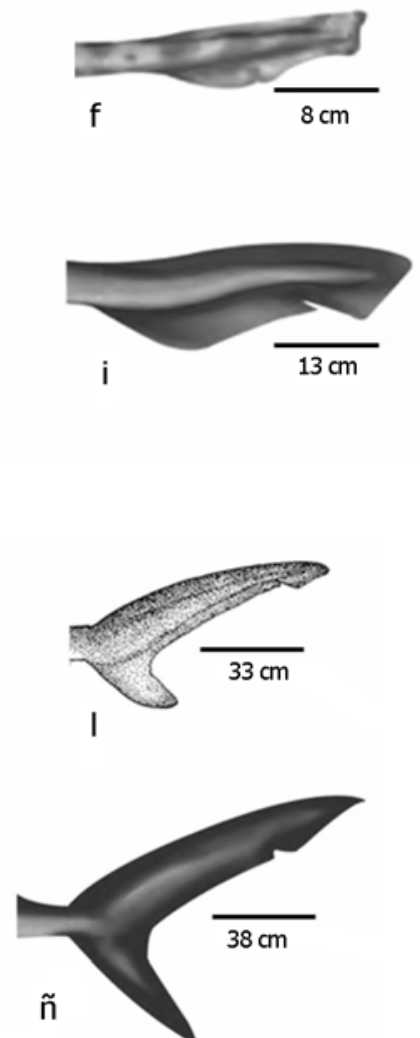

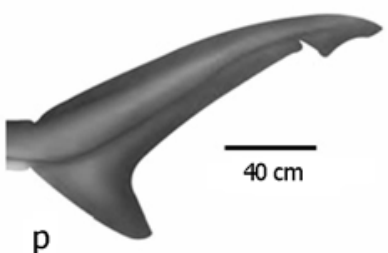

Figura 4. Ilustración de la aleta caudal de Carcharhiniformes (a-p), indicando longitud corporal total (LT) de cada especie / Illustration of the caudal fin Carcharhiniformes (a-p), indicating the total body length (TL) of each species Nombres científicos / Scientific names:

a) SCYLIORHINIDAE: Apristurus brunneus (Gilbert, 1892) LT = $69 \mathrm{~cm}$; b) Apristurus nasutus De Buen, $1959 \mathrm{LT}=56 \mathrm{~cm}$; c) Bythaelurus canescens (Günther, 1878) LT = $70 \mathrm{~cm}$; d) Cephaloscyllium ventriosum (Garman, 1880) LT = $100 \mathrm{~cm}$, e) Cephalurus cephalus Gilbert, $1892 \mathrm{LT}=28 \mathrm{~cm}$; f) Schroederichthys bivius (Müller \& Henle, 1838) LT = $82 \mathrm{~cm}$; g) Schroederichthys chilensis (Guichenot, 1848) LT = 62 cm; h) TRIAKIDAE: Galeorhinus galeus (Linnaeus, 1758) LT = $195 \mathrm{~cm}$; i) Mustelus mento Cope, $1877 \mathrm{LT}=130 \mathrm{~cm}$, j) Mustelus whitneyi Chirichigno, $1973 \mathrm{LT}=87$ $\mathrm{cm}$; k) Triakis maculata Kner \& Steindachner, $1867 \mathrm{LT}=240 \mathrm{~cm}$; I) CARCHARHINIDAE: Carcharhinus falciformis (Müller \& Henle 1839) LT = $330 \mathrm{~cm} ; \mathrm{m}$ ) Carcharhinus galapagensis (Snodgrass \& Heller, 1905) LT = $370 \mathrm{~cm} ; \mathrm{n}$ ) Carcharhinus obscurus (Lesueur 1818$) \mathrm{LT}=400 \mathrm{~cm}, \tilde{\mathrm{n}})$ Prionace glauca (Linnaeus, 1758) LT = $380 \mathrm{~cm}$; o) SPHYRNIDAE: Sphyrna lewini (Griffith \& Smith, 1834) LT = 470 cm; p) Sphyrna zygaena (Linnaeus, 1758) LT $=400 \mathrm{~cm}$ 
Clave 4. Clave taxonómica para la determinación de familias, géneros y especies del orden de Squaliformes

1 (2) Margen terminal escasamente desarrollado o ausente ....................................................................... 3

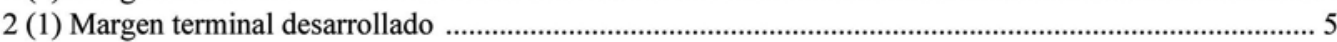

3 (4) Margen dorsal recto ................................................................................................. Squalidae: 11

4 (3) Margen dorsal redondeado Echinorhinidae: Margen postventral superior redondeado. Extremo ventral redondeado. Margen terminal poco desarrollado. Ángulo de inclinación de la aleta caudal de $20^{\circ}$

. Echinorhinus cookei (Fig. 5 g).

5 (6) Margen subterminal amplio o escasamente desarrollado

Echinorhinus cookei (Fig. $5 \mathrm{~g})$.

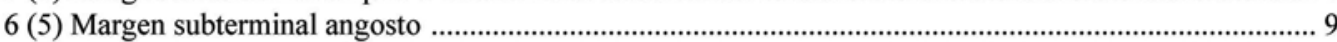

7 (8) Lóbulo ventral corto. Ángulo de inclinación de la aleta caudal entre $13^{\circ}$ y $27^{\circ}$............. Etmopteridae: 15

8 (7) Lóbulo ventral alargado. Ángulo de inclinación de la aleta caudal entre $22^{\circ}$ y $50^{\circ}$.

Somniosidae: 37

9 (10) Margen postventral superior corto

Dalatiidae: 46

10 (9) Margen postventral superior alargado

Centrophoridae: 52

11 (12) Extremo posterior del lóbulo dorsal en punta. Lóbulo terminal angosto, muesca posterior aguzada y margen posterior ventral inferior recto. Ángulo de inclinación de la aleta caudal de $35^{\circ}$

Squalus mitsukurii (Fig. $5 \mathrm{z}$ ).

12 (11) Extremo posterior del lóbulo dorsal redondeado. Lóbulo terminal ancho, muesca posterior redondeada y margen posterior ventral inferior redondeado. Ángulo de inclinación de la aleta caudal entre $24^{\circ}$ y $45^{\circ} \ldots . .13$ 13 (14) Margen postventral superior redondeado. Sin margen terminal. Lóbulo ventral ancho, muesca posterior redondeada. Ángulo de inclinación de la aleta caudal de $45^{\circ}$ Squalus blainville (Fig. 5 y). 14 (13) Margen post ventral superior recto. Con margen terminal. Lóbulo ventral angosto, muesca posterior en forma de "C". Ángulo de inclinación de la aleta caudal de $24^{\circ}$ Squalus acanthias (Fig. $5 \mathrm{x}$ ).

15 (16) Sin margen postventral inferior o muy poco desarrollado. Ángulo de inclinación de la aleta caudal entre $13^{\circ}$ y $26^{\circ}$ Aculeola, Centroscyllium: 17

16 (15) Con margen postventral inferior. Ángulo de inclinación de la aleta caudal entre $12^{\circ}$ y $50^{\circ}$

Etmopterus: 20

17 (18) Extremo ventral redondeado. Margen terminal y margen subterminal angosto. Ảngulo de inclinación de la aleta caudal de $13^{\circ}$ Aculeola nigra (Fig. 5 h).

18 (17) Extremo ventral en punta. Con o sin márgenes terminal y subterminal. Ángulo de inclinación de la aleta caudal mayor a $13^{\circ}$ Centroscyllium: 19

19 (20) Muesca posterior ausente

Etmopterus: 23

21 (22) Margen terminal redondeado. Ángulo de inclinación de la aleta caudal de $19^{\circ}$

22 (21) Margen terminal recto. Ángulo de inclinación de la aleta caudal de $26^{\circ}$

C. granulatum (Fig. 5 i).

22 (21)

23 (24) Margen post ventral superior ondulado .......................................................................................... 25

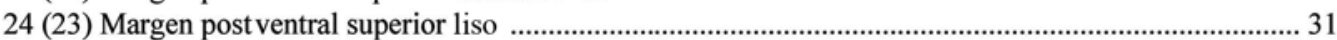

25 (26) Extremo posterior con una mancha blanca. Muesca posterior estrecha, margen subterminal recto. Ángulo de inclinación de la aleta caudal de $26^{\circ}$ Etmopterus pycnolepis (Fig. 5 ñ).

26 (25) Extremo posterior sin una mancha blanca ..................................................................................... 27

27 (28) Con muesca subterminal. Muesca posterior notoria, margen subterminal oblicuo. Ángulo de inclinación de la aleta caudal de $13^{\circ}$........................................................... Etmopterus pusillus (Fig. 5 o).

28 (27) Sin muesca subterminal. Muesca posterior notoria o incipiente. Ángulo de inclinación de la aleta caudal mayor que $14^{\circ}$

29 (30) Margen preventral redondeado. Ángulo de inclinación de la aleta caudal de $19^{\circ}$

Etmopterus unicolor (Fig. 5 p).

30 (29) Margen preventral recto. Angulo de inclinación de la aleta caudal de $17^{\circ}$... Etmopterus lucifer (Fig. $5 \mathrm{n}$ ). 31 (32) Extremo ventral redondeado. Ángulo de inclinación de la aleta caudal de $24^{\circ}$

Etmopterus litvinovi (Fig. $5 \mathrm{~m}$ ).

32 (31) Extremo ventral redondeado o en punta. Ángulo de inclinación de la aleta caudal menor a $24^{\circ}$..........33 
33 (34) Margen terminal recto y oblicuo. Margen subterminal ancho. Ángulo de inclinación de la aleta caudal de $12^{\circ}$ Etmopterus brachyurus (Fig. 5 k).

34 (33) Margen terminal redondeado. Margen subterminal angosto. Ángulo de inclinación de la aleta caudal mayor a $12^{\circ}$ 35

35 (36) Margen subterminal contenido cuatro veces en el margen postventral superior. Extremo posterior recto. Ángulo de inclinación de la aleta caudal de $16^{\circ}$

Etmopterus granulosus (Fig. 5 1). 36 (35) Margen subterminal contenido dos veces en el margen postventral superior. Extremo posterior dirigido hacia abajo. Ángulo de inclinación de la aleta caudal de $14^{\circ}$ Etmopterus villosus (Fig. 5 q). 37 (38) Margen postventral superior casi del mismo tamaño que el margen terminal. Ángulo de inclinación de la aleta caudal menor a $50^{\circ}$

38 (37) Margen postventral superior más pequeño que el margen terminal. Ángulo de inclinación de la aleta caudal de $50^{\circ}$ 39 (40) Margen terminal redondeado. Ángulo de inclinación de la aleta caudal de $30^{\circ}$ Somniosus pacificus (Fig. $5 \mathrm{w}$ ).

40 (39) Margen terminal recto u oblicuo. Ángulo de inclinación menor a $30^{\circ}$

Scymnodalatias oligodon (Fig. $5 \mathrm{u}$ ).

41 (42) Muesca posterior presente. Ángulo de inclinación de la aleta caudal de $12^{\circ}$

Zameus squamulosus (Fig. 5 v)

42 (41) Muesca posterior presente o ausente Centroscymmus: 43

43 (44) Lóbulo ventral angosto, margen preventral recto. Presencia de una pequeña muesca en el extremo posterior. Margen post ventral superior ondulado. Ángulo de inclinación de $22^{\circ}$

Centroscymnus crepidater (Fig. 5 r).

44 (43) Margen postventral superior redondeado, margen subterminal amplio (casi el doble que el margen terminal). Ángulo de inclinación de la aleta caudal de $24^{\circ}$................ Centroscymnus macracanthus (Fig. $5 \mathrm{~s}$ ). 45 (46) Margen postventral superior recto, margen subterminal angosto (menos del doble que el margen terminal). Ángulo de inclinación de la aleta caudal de $26^{\circ}$ Centroscymnus owstonii (Fig. 5 t). 46 (47) Margen terminal liso; margen postventral superior liso. Ángulo de inclinación de la aleta caudal menor a $40^{\circ}$ 48 47 (46) Margen terminal ondulado; margen postventral superior ondulado. Ángulo de inclinación de la aleta caudal de $40^{\circ}$ Isistius brasiliensis (Fig. 5 e).

48 (49) Margen subterminal contenido cuatro veces en el margen terminal, muesca posterior poco desarrollada. Ảngulo de inclinación de $35^{\circ}$ Euprotomicrus bispinatus (Fig. 5 c). 49 (48) Margen subterminal contenido menos de cuatro veces en el margen terminal, muesca posterior notoria. Ángulo de inclinación menor a $35^{\circ}$ 50 50 (51) Margen terminal recto, margen preventral redondeado. Ángulo de inclinación de la aleta caudal de $26^{\circ}$ Heteroscymnoides marleyi (Fig. 5 d). 51 (50) Margen terminal redondeado, margen preventral recto. Ángulo de inclinación de la aleta caudal de $31^{\circ}$ Mollisquama parini (Fig. $5 \mathrm{f}$ )

52 (53) Sin muesca posterior, con muesca subterminal, lóbulo ventral ancho, extremo ventral en punta. Ángulo de inclinación de la aleta caudal de $32^{\circ}$ Centrophorus squamosus (Fig. 5 a). 53 (52) Con muesca posterior, sin muesca subterminal, lóbulo ventral angosto, extremo ventral redondeado. Ángulo de inclinación de la aleta caudal de $25^{\circ}$ Deania calcea (Fig. 5 b). 

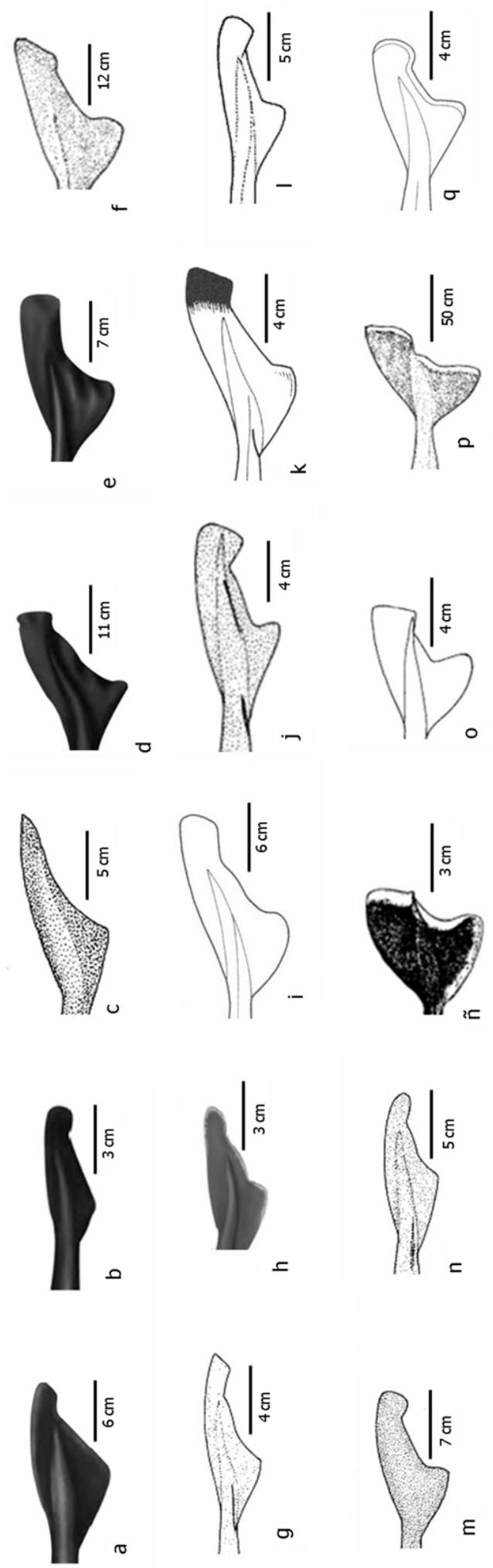
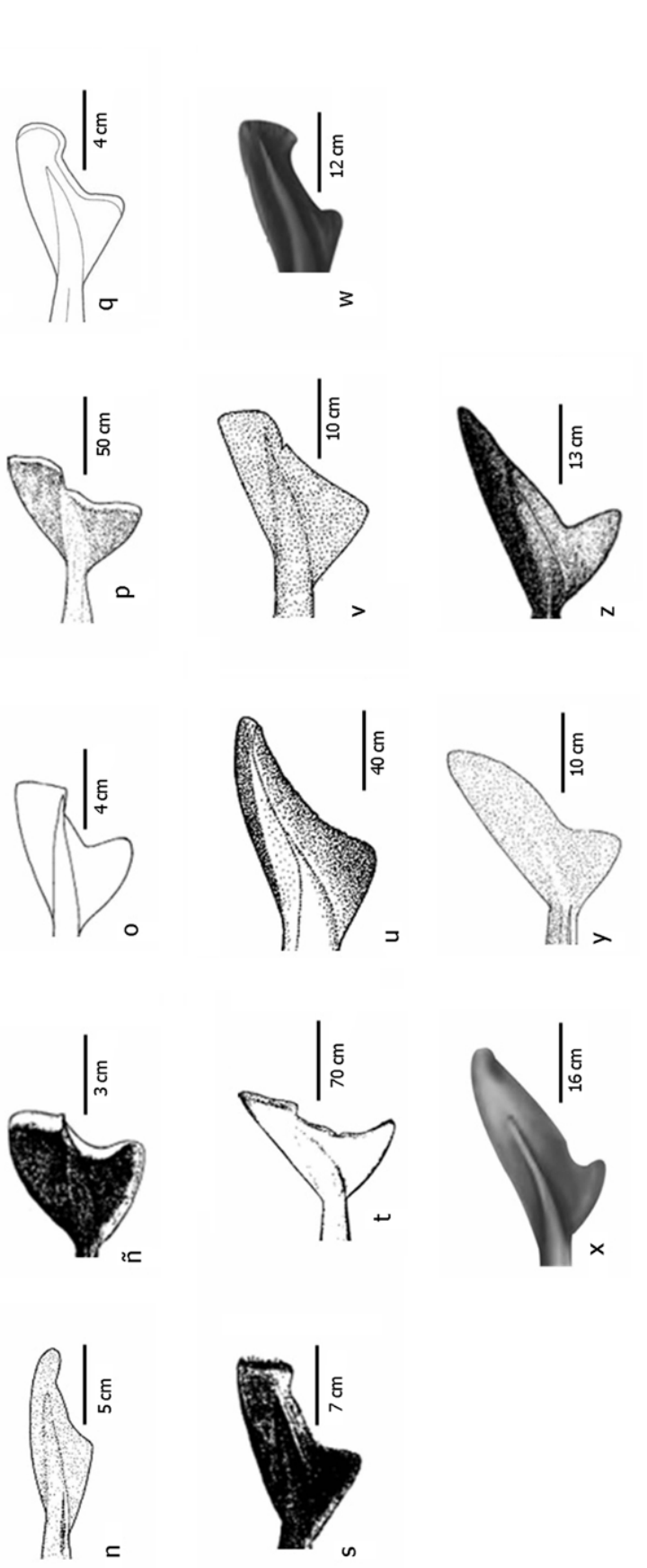

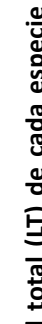

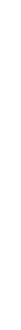
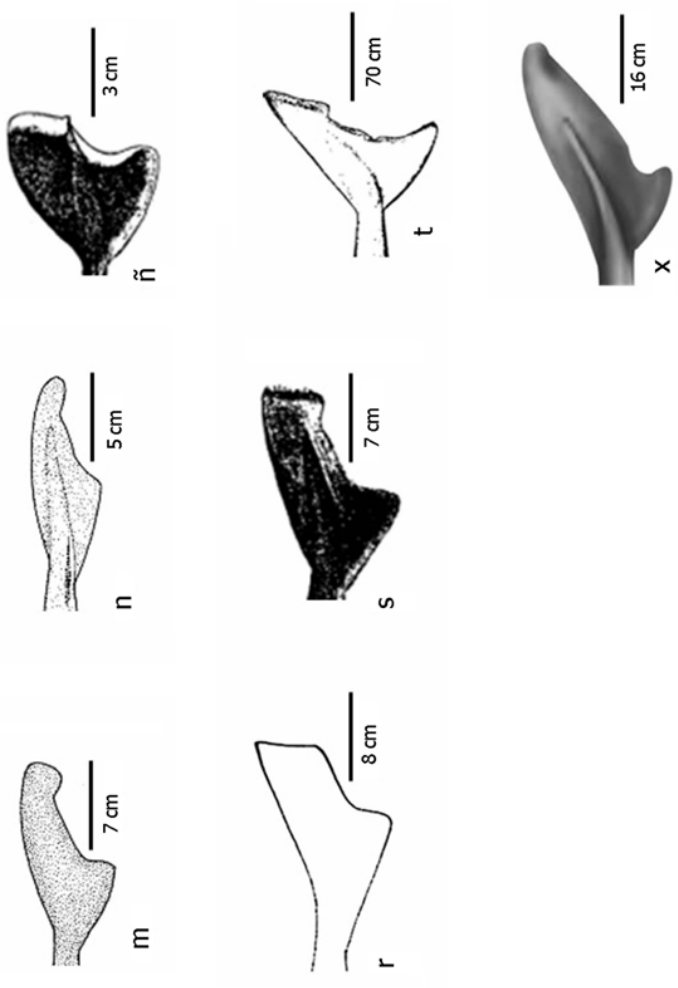

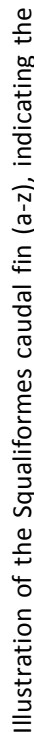

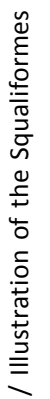

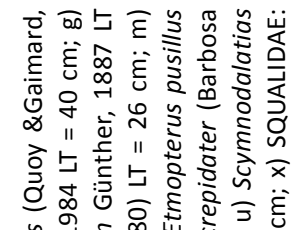

足

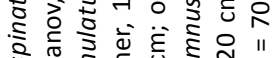

资

รูำ कह

है

응 仓ิे 옹

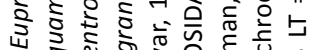

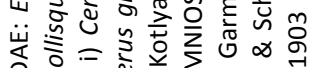

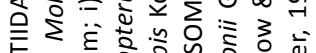

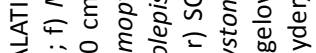

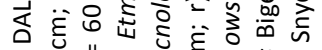
o 品 = हี

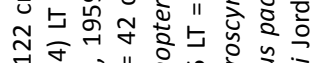

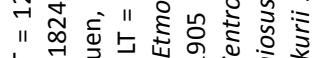
曰

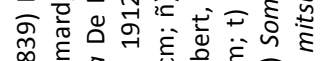

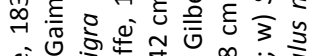

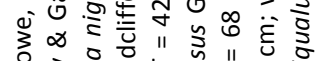

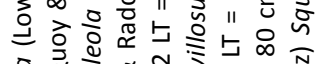
ชั

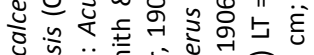

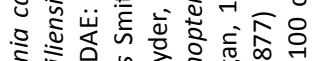

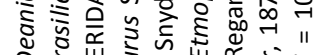

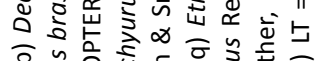

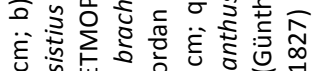

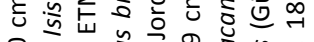

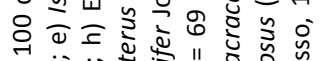
"

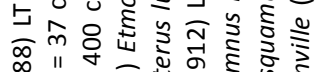
草 ง

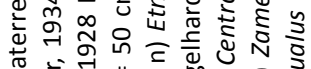

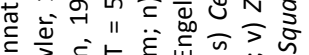

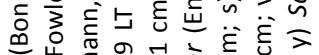
s 仓े نे

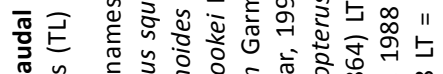

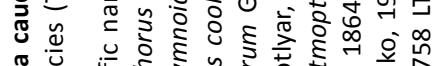

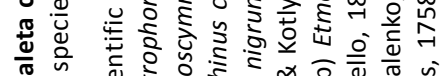
क人

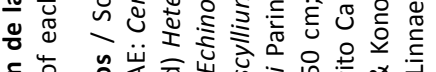

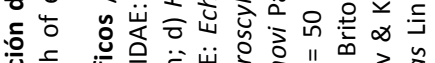

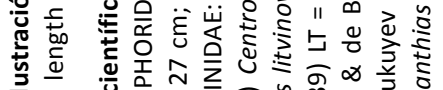
点㐫

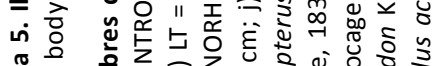

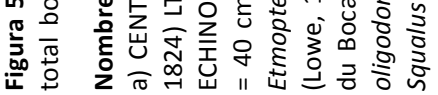




\section{Discusión}

Los diferentes componentes morfológicos de la aleta caudal de los tiburones ofrecen en su conjunto una valiosa información para la determinación taxonómica de familias, géneros y especies. Las claves taxonómicas de condrictios generalmente no consideran en detalle aspectos de la morfología de la aleta caudal de los tiburones, por lo que se consideraron aquellos caracteres fáciles de observar y con mayor capacidad discriminante para clasificar los grupos taxonómicos.

Para elaborar esta clave se distinguen caracteres tales como: elevación o declinación del margen dorsal (ángulo de inclinación), forma del margen terminal (recto, redondeado), extensión del lóbulo dorsal, presencia o ausencia de muesca subterminal o posterior, entre otros. En el caso particular de la elevación de la aleta caudal, existiría una leve variación en los ángulos medidos de cada una de las especies (no más de uno o dos grados), esto debido a la variación intraespecífica producto de factores no biológicos como: error en la medición del ángulo de la aleta caudal en el registro fotográfico o dibujo y de factores biológicos tales como el estado de madurez y desarrollo ontogenético (Reiss \& Bonnan 2010). Tales variaciones son en condiciones controladas como en terreno, de laboratorio, puertos de desembarque, embarcaciones, etc., sin embargo, es un carácter confiable que facilita la diferenciación de las especies. Así, se puede afirmar que los caracteres seleccionados son de utilidad para la determinación taxonómica, esto debido a su fácil observación, invariables en el tiempo y, además, de ser caracteres conservadores de grupos taxonómicos (órdenes, familias, géneros, especies). Sumado a esto, la aleta caudal es una estructura de fácil conservación en colecciones ictiológicas, por lo que teniendo la diagnosis clara de un espécimen, es una buena alternativa para las colecciones ictiológicas o museos por su comodidad para guardarlas, en comparación con el almacenamiento del animal completo. Sin embargo, pese a todas las ventajas que tienen los distintos componentes de la aleta caudal, éstos no han sido considerados en su totalidad como caracteres taxonómicos en las diferentes claves que existen para este grupo de peces. En este sentido, Hernández et al. (2009) han sido una excepción, puesto que estos autores emplearon la aleta caudal (considerando además otros elementos) enfocados en la determinación de la aleta caudal de algunas especies involucradas en el comercio de aletas, restringiéndose así a unas pocas especies de interés comercial como son: Prionace glauca, Isurus oxyrhinchus y Lamna nasus. Así mismo, Sebastian et al. (2008) y Hernández et al. (2010), obtuvieron la caracterización de algunas especies pelágicas, en las costas de Chile mediante la determinación genética de las aletas de tiburones, incluyendo la caudal.

Por otro lado, se han adicionado a la clave dos especies que se han registrado en aguas chilenas, Carcharhinus obscurus y C. falciformis, siendo que a ambas especies por años se les consideraba dudosa su aparición en Chile (Pequeño 1989, Lamilla \& Bustamante 2005). Sin embargo, tanto para C. obscurus como para C. falciformis, mediante nuevos antecedentes bibliográficos, se puede confirmar su presencia en el centro norte de Chile, aumentando las especies chilenas de 56 (Sáez et al. 2010) a 58 especies (Sebastian et al. 2008, FISHWISE 2011²).

Los peces constituyen un recurso natural renovable muy preciado por el hombre. La Food and Agricultural Organization (FAO), está preocupada del uso de los peces a través de la pesca. Esto implica que se debe conocer las especies, su estado de conservación, características biológicas, etc. y esto último no se logra si no se tiene la capacidad para determinar la identidad de cada individuo capturado. Debido a que, muchas veces hay tiburones que han sido parcialmente destrozados durante el proceso de captura, existe la posibilidad de recurrir a partes del organismo no descartados, para emitir un juicio de la determinación de la especie. De esta manera, se espera fomentar el uso de las partes que componen la aleta caudal para ser empleadas y valoradas como una nueva herramienta taxonómica, que puede ser usada aisladamente o como complemento de los caracteres taxonómicos comúnmente más conocidos y empleados en las distintas claves taxonómicas que existen en la literatura.

\section{Agradecimientos}

Los autores agradecen el financiamiento parcial al proyecto Fondo de Innovación Competitiva Regional FICR/DID UACH 2010 'Innovación y sustentabilidad bioeconómica en la pesquería artesanal de pequeños peces pelágicos en la Región de Los Ríos’. Gobierno Regional de Los Ríos, y a la dibujante Judeh Elalam por la elaboración de algunas ilustraciones de esta publicación.

${ }^{2}$ FISHWISE 2011. Universal Fish Catalogue [on line] <www.fishwise.co.za> 
A Hernán Flores (Biólogo Marino, Laboratorio de Elasmobranquios (ELASMOLAB), Instituto de Ciencias Marinas y Limnológicas, Universidad Austral de Chile) por la preparación del material usado en el trabajo.

\section{LITERATURA CITADA}

Aalbers A, D Bernal \& CA Sepúlveda. 2010. The functional role of the caudal fin in the feeding ecology of the common thresher shark Alopias vulpinus. Journal of Fish Biology 73: 1863-1868.

Brito JL. 2004a. Hallazgo de Somniosus pacificus Bigelow \& Schroeder, 1944 (Squaliformes: Squalidae) en San Antonio, Chile central. Investigaciones Marinas 32(2): 137-139.

Brito JL. 2004b. Presencia del tiburón martillo Sphyrna zygaena (Carcharhiniformes: Sphyrnidae) y nuevo registro del tiburón espinudo Echinorhinus cookei (Squaliformes: Squalidae) en San Antonio, Chile central. Investigaciones Marinas 32(2): 141-144.

Compagno LJV. 1984a. FAO Species Catalogue. Part 1.Sharks of the world. An annotated and illustrated catalogue of shark species known to date. Hexanchiformes to Lamniformes. FAO Fisheries Synopsis 125 4(1): 1-249.

Compagno LJV. 1984b. FAO Species Catalogue. Part 2. Sharks of the world. An annotated and illustrated catalogue of shark species known to date. Carcharhiniformes. FAO Fisheries Synopsis 125 4(2): 251-655.

Compagno LJV. 1999. Systematics and body form. In: Hamlett WC (ed). Sharks, skates and rays. The biology of elasmobranch fishes, pp. 1-42. Johns Hopkins University Press, Baltimore.

Compagno LJV, D Dando \& S Fowler. 2005. A field guide to the sharks of the world, 368 pp. Harper Collins Publishing, London.

Hernández S, PA Haye \& E Acuña. 2009. Identificación morfológica de las aletas de los principales tiburones pelágicos comercializados en Chile: Azulejo (Prionace glauca Linnaeus), Marrajo (Isurus oxyrhynchus Rafinesque), y Tintorera (Lamna nasus Bonnaterre). Gayana 73(1): 33-39.
Hernández S, C Gallardo-Escárate, J Alvares-Borego, MT González \& PA Haye. 2010. A multidisciplinary approach to identify pelagic shark fins by molecular, morphometric and digital correlation data. Hidrobiológica 20(1): 71-80.

Lamilla J \& C Bustamante. 2005. Guía para el reconocimiento de tiburones, rayas y quimeras de Chile. Oceana 17: 1-80.

Lloris D \& J Rucabado. 1991. Ictiofauna del canal Beagle (Tierra del Fuego), aspectos ecológicos y análisis biogeográfico. Publicación Especial, Instituto Español de Oceanografía 8: 1-182.

Pequeño G. 1977. El género Galeorhinus en Chile (Elasmobranchii: Triakidae). Revista de Biología Marina 16(2): 183-188.

Pequeño G. 1979a. El género Notorhynchus en Chile (Elasmobranchii). Revista de Biología Marina 16(3): 257264

Pequeño G. 1979b. Nota sobre un ejemplar de Cetorhinus maximus (Gunnerus, 1765) capturado frente a Corral, Chile. Neotropica 73: 97-98.

Pequeño G. 1989. Peces de Chile, lista sistemática revisada y comentada. Revista de Biología Marina 24(2): 1-132.

Reiss K \& MF Bonnan. 2010. Ontogenetic scaling of caudal fin shape in Squalus acanthias (Chondrichthyes, Elasmobranchii): A geometric morphometric analysis with implications for caudal fin functional morphology. The Anatomical Record 293: 1184-1191.

Sáez S, G Pequeño \& J Lamilla. 2010. Clave taxonómica del Superorden Squalomorphi de Chile (Pisces: Elasmobranchii). Revista de Biología Marina y Oceanografía 45 (S1): 619634.

Sebastian H, PA Haye \& MS Shivji. 2008. Characterization of the pelagic shark-fin trade in north-central Chile by genetic identification and trader surveys. Journal of Fish Biology 73: 2293-2304.

Stewart K \& DF Simaner. 1977. Body form and locomotion in sharks. American Zoologist 17: 343-354.

Valenzuela A, C Bustamante \& J Lamilla. 2008. Morphological characteristics of five bycatch sharks caught by southern Chilean demersal longline fisheries. Scientia Marina 72(2): 231-237.

Recibido el 23 de enero de 2012 y aceptado el 7 de junio de 2012

Editor Asociado: Mauricio Landaeta D. 